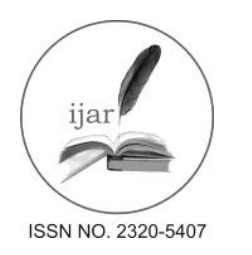

Journal homepage:http://www.journalijar.com
Journal DOI:10.21474/IJAR01

RESEARCH ARTICLE

\title{
COMPARATIVE EVALUATION OF FLUORIDE UPTAKE BY DENTINE FROM GLASS IONOMER CEMENT FUNCTIONALIZED WITH CHLORHEXIDINE HEXAMETAPHOSPHATE NANOPARTICLES AND TYPE II GIC AT 3 DAYS- IN VITRO STUDY.
}

INTERNATIONAL JOURNAL

OF ADVANCED RESEARCH

\section{Tanvi Satpute ${ }^{1}$, MDS,Sanjyot Mulay ${ }^{2}$, MDS.}

1. PG student, Department of Conservative Dentistry and Endodontics, Dr. D. Y. Patil Dental College and Hospital, Pimpri, Pune.

2. Professor and HOD, Department of Conservative Dentistry and Endodontics, Dr. D. Y. Patil Dental College and Hospital, Pimpri, Pune.

\section{Manuscript Info}

Manuscript History:

Received: 16 March 2016

Final Accepted: 19 April 2016

Published Online: May 2016

Key words:

GIC; Chlorhexidine; nanoparticles; fluoride uptake

*Corresponding Author

TanviSatpute.

\begin{abstract}
Aim: To evaluate and compare the fluoride uptake by dentin from Glass ionomer cement functionalized with ChlorhexidineHexametaphosphate nanoparticles and Type II GIC.

Methodology: Nine human premolars were used on which Class I cavities measuring $5 \mathrm{~mm} \times 3 \mathrm{~mm} \times 2 \mathrm{~mm}$ were prepared using round and straight diamond abrasive. Specimens were divided into three groups. First group of teeth specimens $(n=3)$ were filled with GIC containing novel antimicrobial nanoparticles composed of (CHX-HMP) ChlorhexidineHexametaphosphate. Second group of teeth specimens $(n=3)$ were filled with Type II GIC .The third group of specimens $(n=3)$ served as control where no restorative material was filled. The samples were kept at $37{ }^{\circ} \mathrm{C}$ and $100 \%$ humidity for 3 days. Each tooth was sectioned into two half's mesio-distally using diamond disc. Teeth sections were evaluated for fluoride uptake by dentin under SEM using EDAX image analyzer software. The results were tabulated and analyzed.
\end{abstract}

Result: The amount of fluoride present in Group I (Type II GIC) was in the range of 7.64 atomic wt \% and Group II (CHXHMP-GIC) was 6.71 atomic $\mathrm{wt} \%$.Flouride was detected till depths of $200 \mu \mathrm{m}$ for Type II restorative GIC

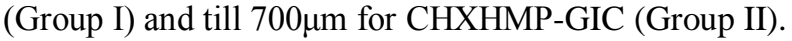

Conclusion: Fluoride release and consequent uptake by dentine was not significantly affected by the substitution of antimicrobial nanoparticles. The fluoride penetration and its effect on enhancing remineralization with the added antimicrobial effect of CHX is promising.

Copy Right, IJAR, 2016.. All rights reserved.

\section{Introduction:-}

In modern dentistry, Glass ionomer cements (GICs) are a class of biomaterials having widespread application especially in Minimally intervention dentistry (MID) for the treatment of dental caries. ${ }^{1,2}$

The therapeutic procedures used in the treatment of caries may not always eliminate all the microorganisms in the residual tissues. The persisting cariogenic bacteria, with the lack of hermetic seal, can cause recurrent caries, leading to failure of restoration. ${ }^{3}$ 
Also changing concepts using Atraumatic Restorative technique involves removal of infected, disorganized dentin and preservation of the affected less organized dentin that has the potential to remineralize.

The use of GIC is extremely popular in dental practice, due to its properties of chemical bonding to mineralized tooth structures, coefficient of heat expansion similar to that of dentin, adequate biocompatibility and most importantly fluoride ion release, which contribute to the remineralization process. ${ }^{4,5}$

GIC which offers an additional antimicrobial and antibiofilm efficacy would be of considerable clinical benefit. Such a material could reduce recurrent decay in the vicinity of a restoration and could provide an antibacterial seal under other materials, protecting the pulp from bacterial ingress. ${ }^{6}$

A recent publication in 2014 describes surface functionalization of materials using Chlorhexidine Hexametaphosphate (CHX-HMP) nano particles and it was found that they acted as slow release devices for soluble chlorhexidine (CHX) which is a potent antimicrobial agent in widespread use in medicine and dentistry. ${ }^{7}$

The amount of fluoride uptake by dentin has been found to vary with different parameters like structure of the set cement, setting reaction, composition, different time intervals etc. There are few studies on the amount of fluoride uptake by dentin from GIC but no documented literature on fluoride uptake by dentin from GIC functionalized with CHX.

Thus the aim of this study was to evaluate and compare the fluoride uptake by dentin from Glass ionomer cement functionalized with Chlorhexidine Hexametaphosphate nano particles (CHXHMP-GIC) and Type II GIC.

\section{Materials and method:-}

\section{Selection and Preparation of Teeth:-}

Nine sound extracted human teeth were obtained from the Department of Oral surgery at Dr. D.Y Patil Dental College and Hospital. After the removal of tissue remainders, prophylaxis and washing, only teeth without anatomical and structural defects were selected. These were stored in saline.

\section{Preparation of nanoparticles:-}

Chlorhexidinedigluconate (Dentochlor, Ammdent) was mixed with Sodium Hexametaphosphate (Thomas Baker) in deionized water to obtain a final concentration of $4 \mathrm{mM} \mathrm{CHX}$ and $5 \mathrm{mM}$ HMP. The resulting colloidal suspension of CHX-HMP nanoparticles was mixed thoroughly and then centrifuged at $10000 \mathrm{rpm}$ for $60 \mathrm{~min}$. (Figure 1 and 2)

The supernatant was removed and discarded. The nanoparticle sediment was dried in an incubator for $48 \mathrm{~h}$ at $40^{\circ} \mathrm{C}$. The sediment composed of nanoparticle aggregates was removed from the centrifuge tubes (Figure 3) and ground to a fine white powder using an agate mortar and pestle.

The nanoparticle powder created by grinding the nanoparticle pellet was used to substitute for the GIC powder at fraction of $2 \%$ by mass.

\section{Preparation of samples:-}

Class I cavities measuring $5 \mathrm{~mm} \times 3 \mathrm{~mm} \times 2 \mathrm{~mm}$ were prepared using round and straight diamond abrasive points.

Teeth were randomly divided into three groups. Group I specimens were restored with Type II GIC ( $\mathrm{n}=3$ teeth/ 6 sections), Group II specimens were restored with (GIC CHX-HMP) ( $n=3$ teeth/ 6 sections) and Group III specimens served as control, specimens were not restored. ( $\mathrm{n}=3$ teeth/ 6 sections).

\section{Testing:-}

The samples were kept at $37{ }^{\circ} \mathrm{C}$ and $100 \%$ humidity for 3 days then were sectioned and analyzed. Each tooth was sectioned into two half's mesio-distally using diamond disc. Determination of the chemical element was done using SEM with EDAX software. The depths were then analyzed by SEM at X100 magnification operated at $20.0 \mathrm{kV}$ and EDS. 


\section{Results:-}

The qualitative analysis showed that the intensity of fluoride signals for both Group I and II was similar. The mean amount of fluoride present in Group I (Type II GIC) was 7.64 atomic wt \% and Group II (CHXHMP-GIC) was 6.71 atomic wt $\%$. The EDAX software highlighted areas of dentin with fluoride depth (penetration). The depths were then analyzed by SEM at X100 magnification operated at $20.0 \mathrm{kV}$ and EDS. Flouride was detected till depths of 200 $\mu \mathrm{m}$ for Type II restorative GIC (Group I) and till 700 $\mu \mathrm{m}$ for CHXHMP-GIC (Group II).

\section{Discussion:-}

Glass ionomer cements (GICs) are a class of biomaterials used in modern dentistry. It has been used for more than 20 years and is considered to be a fluoride reservoir. ${ }^{8}$ Its ability to inhibit recurrent caries formation is one of its important clinical property. ${ }^{9}$ GICs have several favorable properties which make them suitable for a multitude of applications including for filling cavities caused by tooth decay or wear, as cavity liners, as fissure sealants, and as cements to form an adhesive bond between the tooth and a prosthetic dental restoration such as a crown or bridge these applications. They are tooth colored and available in a range of shades to allow matching to a patient's natural dentition, they are biocompatible, and they require minimal preparation of the tooth surface prior to application because they inherently adhere to enamel and dentine. ${ }^{7}$

A GIC with additional antimicrobial and antibiofilm efficacy would be of considerable clinical benefit. ${ }^{6}$

The effectiveness of CHX is often related to its property of substantivity due to the ability to bind to anionic substrates, broad spectrum efficacy with rapid onset of action, disruption of the bacterial cell membrane and resultant loss of its intracellular components, bacterial resistance to CHX is also considered unlikely. ${ }^{10,11}$

Chlorhexidine (CHX) has been used in combinationwith GICs, particularly in the forms of digluconate and diacetate. ${ }^{12,13}$ Nevertheless, their antibacterial effect is concentration-dependent, and at high concentrations, CHX may interfere in the physical and mechanical properties of GIC. ${ }^{14}$

Barbour et al (2014) first reported the development of novel antimicrobial nanoparticles (NPs) based on a hexametaphosphate salt of CHX. They found that the NPs adhered rapidly to specimens of glass, titanium, and an elastomeric wound dressing, in a dose-dependent manner. The functionalized materials exhibited a gradual leaching of soluble CHX over a period of at least 50 days. The NP colloid was efficacious against methicillin-resistant Staphylococcus aureus(MRSA) and Pseudomonas aeruginosain both planktonic and biofilm conditions. ${ }^{15}$

Hook et al (2014) subjected the formulation to certain tests and characterized the nanoparticle size, morphology and charge and the release of chlorhexidine and fluoride, tensile strength and morphology of the GIC. They formulated the GIC containing novel antimicrobial nanoparticles composed of chlorhexidinehexametaphosphate at 1, 2, 5, 10 and $20 \%$ powder substitution by mass. They found that the GICs released chlorhexidine, against a wide range of bacteria, over the duration of the experiment in a dose-dependent manner. It was not at the expense of other properties of the material. They also found that the fluoride release was not significantly affected by the substitution of antimicrobial nanoparticles in most formulations and internal structure appeared unaffected up to and including $10 \%$ substitution. Diametral tensile strength decreased numerically with 10 and $20 \%$ substitutions of nanoparticles but the difference was not statistically significant. ${ }^{7}$

The mixing of solutions of CHX and HMP under the conditions described in the above study resulted in the immediate formation of a colloid consisting of particles with maximum average diameter of $150 \mathrm{~nm}$ and an average zeta potential of -45 to $-50 \mathrm{mV}$. The Atomic force microscopy (AFM) and SEM images showed individual nanoparticles and porous nanoparticle (NP) aggregates. Presumably owing the charge of the particles, it resulted in a good colloidal stability at least in part, to the high zeta potential. ${ }^{7}$

So the rationale behind the study was to check the fluoride release of this new material with the addition of CHX nanoparticles and if the subsequent uptake by dentin was significantly affected by the addition of antimicrobial nanoparticles. If the fluoride release remains unaffected, then the material could provide an antibacterial seal under other materials, protecting the pulp from bacterial ingress.

The scanning electron microscope has been used by several authors to assess the demineralisation/remineralisation effect of fluoride-containing products. ${ }^{16}$ In most studies using a scanning electron microscope, to improve image 
quality, samples have been coated with metals such as gold or palladium. ${ }^{17}$ Harding et al. (1994) observed samples using the scanning electron microscope without coating so that they could be observed again, if necessary. In the current study, this technique was used so that there would be an option of reevaluation in the future. ${ }^{18}$

The Energy Dispersive X- Ray analysis (EDAX) system can identify and quantify elements. EDAX identifies elements by energy, using a solid-state detector that discriminates between energies of incoming photons. ${ }^{19}$

There are mechanisms by which fluoride exerts its actions. One of the criteria for evaluation of the cariostatic properties of a restorative material is to determine the fluoride uptake by teeth and its subsequent incorporation into tooth structure. ${ }^{20}$ Studies on the efficacy of fluoride release from dental materials are innumerable, but the amount and depth of fluoride incorporation into dentin are not well-defined.

The clinical use of fluoride to repair early carious lesions is advocated since it enhances enamel remineralization,. When fluoride is present, fluorapatite, forms during the remineralization process. Hydroxyl groups $(\mathrm{OH}-)$ are replaced by Fluoride ions (F-) in the formation of the apatite crystal lattice (Fig. 16). In fact, the presence of fluoride increases the rate of remineralization. ${ }^{21}$

Fluorapatite has been found to be inherently less soluble than hydroxyapatite, even under acidic conditions. When hydroxyapatite dissolves under cariogenic (acidic) conditions, if fluoride is present, then fluorapatite will form. Because fluorapatite is less soluble than hydroxyapatite, it is also more resistant to subsequent demineralization when acid challenged. ${ }^{22}$

The mean amount of fluoride present in Group I (Type II GIC) was 7.64 atomic wt \% and Group II (CHXHMPGIC) was 6.71 atomic wt $\%$. The EDAX software highlighted areas of dentin with fluoride depth (penetration). Flouride was detected till depths of $200 \mu \mathrm{m}$ for Type II restorative GIC (Group I) and till 700 $\mu \mathrm{m}$ for CHXHMP-GIC (Group II). In this study fluoride uptake was not significantly affected while increasing the depth of fluoride penetration into dentin in CHXHMP-GIC (Group II). Matrix Metalloproteinases (MMP's) are indicated as the active proteases that breakdown the collagen fibrils in the hybrid bond layer. There is evidence of the ability of Chlorhexidine to prevent bond deterioration by inhibiting MMP action. ${ }^{23}$

Fluoride evaluation was done at 3 days because literature has documented a "fluoride burst" at 48-72 hrs in conventional GIC (Type II). ${ }^{24}$ Longer evaluation period may be indicated using larger sample size to study the delayed fluoride release from new CHXHMP-GIC nanoparticles.

\section{FIGURES:-}

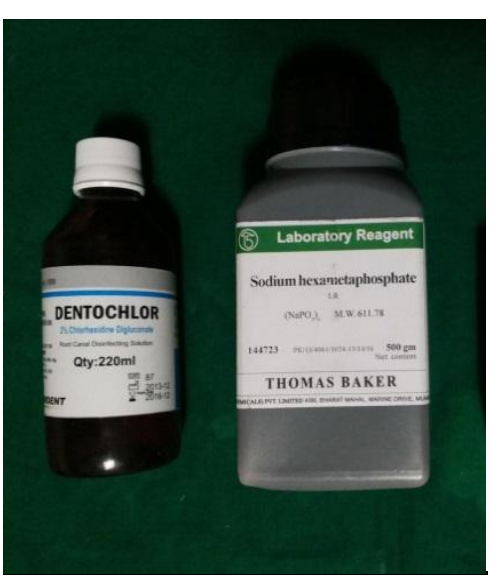

Figure 1:-Dentachlor(Ammdent)And Sodium Hexametaphosphate (Thomas Baker) 

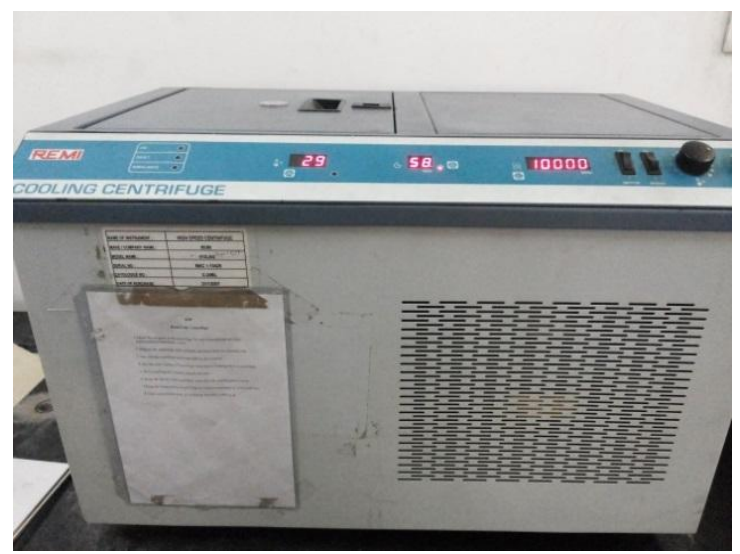

Figure 2. High speed centrifuge (Remi)

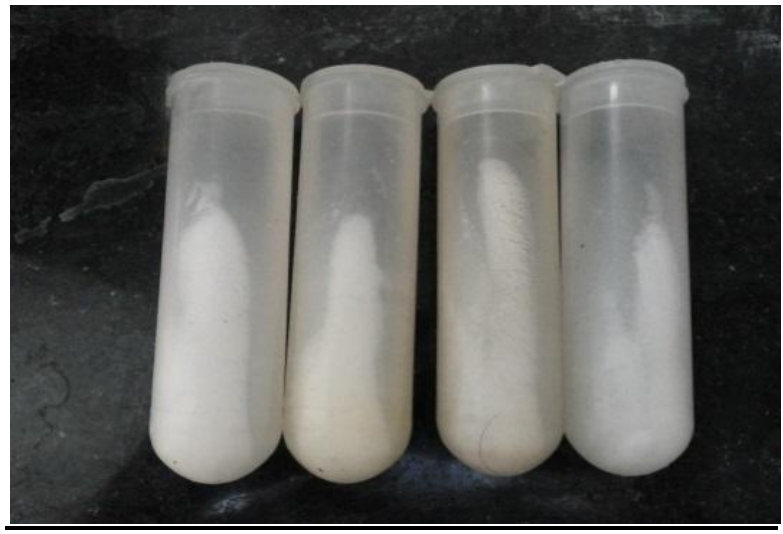

Figure 3. Centrifuge tubeswith nanoparticle sediment

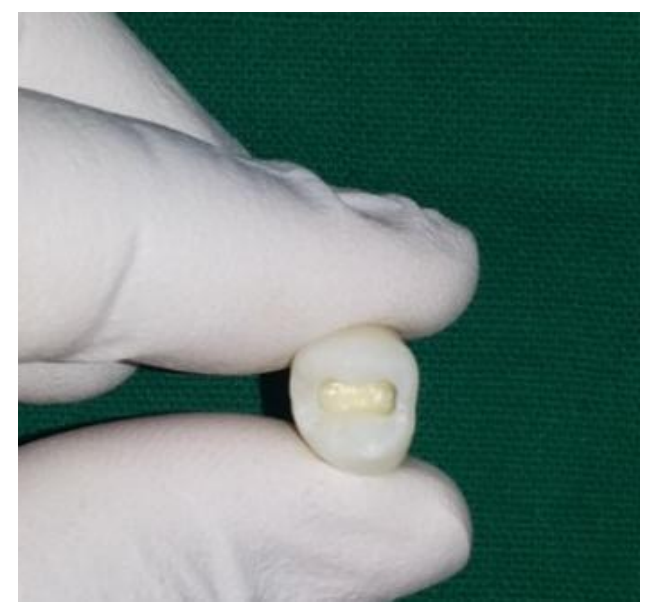

Figure no. 4: Sample 


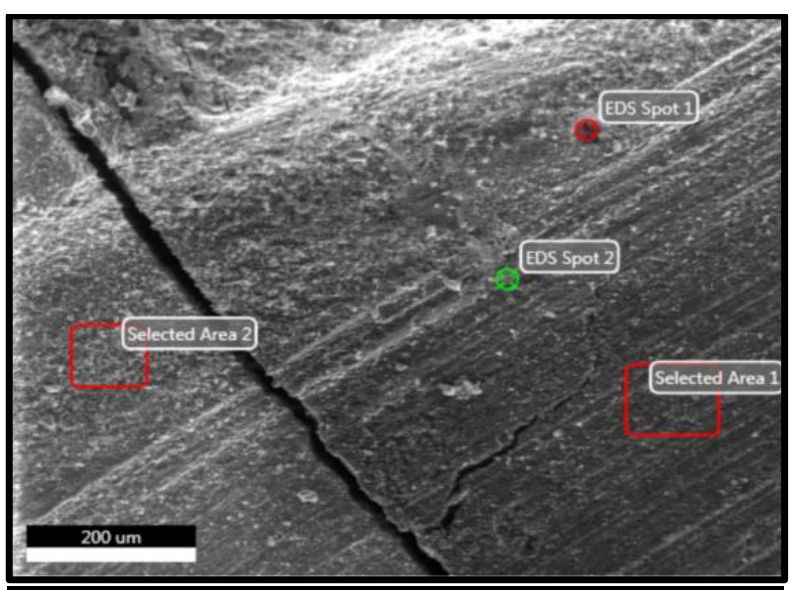

Figure no.5 SEM image at x100 magnification (Group I - Type II GIC)

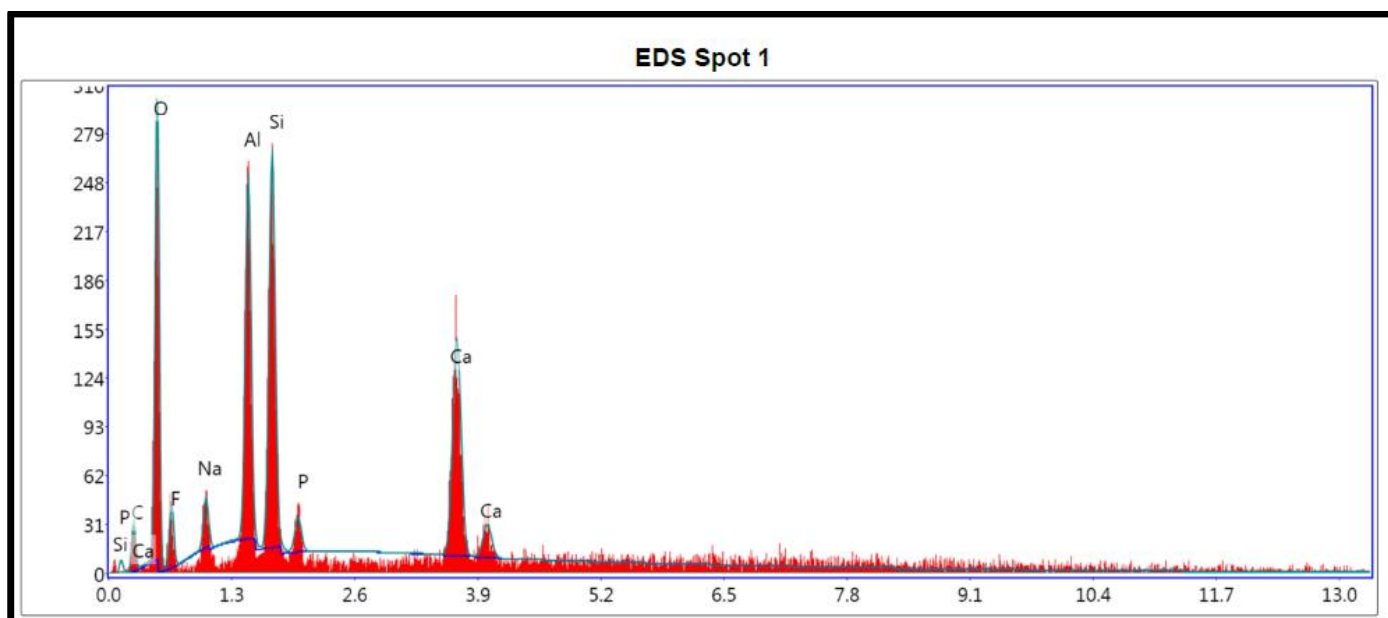

Lsec: 30.00 Cnts 0.000 keV Det: Octane Plus Det

Figure no 6.EDS analysis (Group I - Type II GIC)

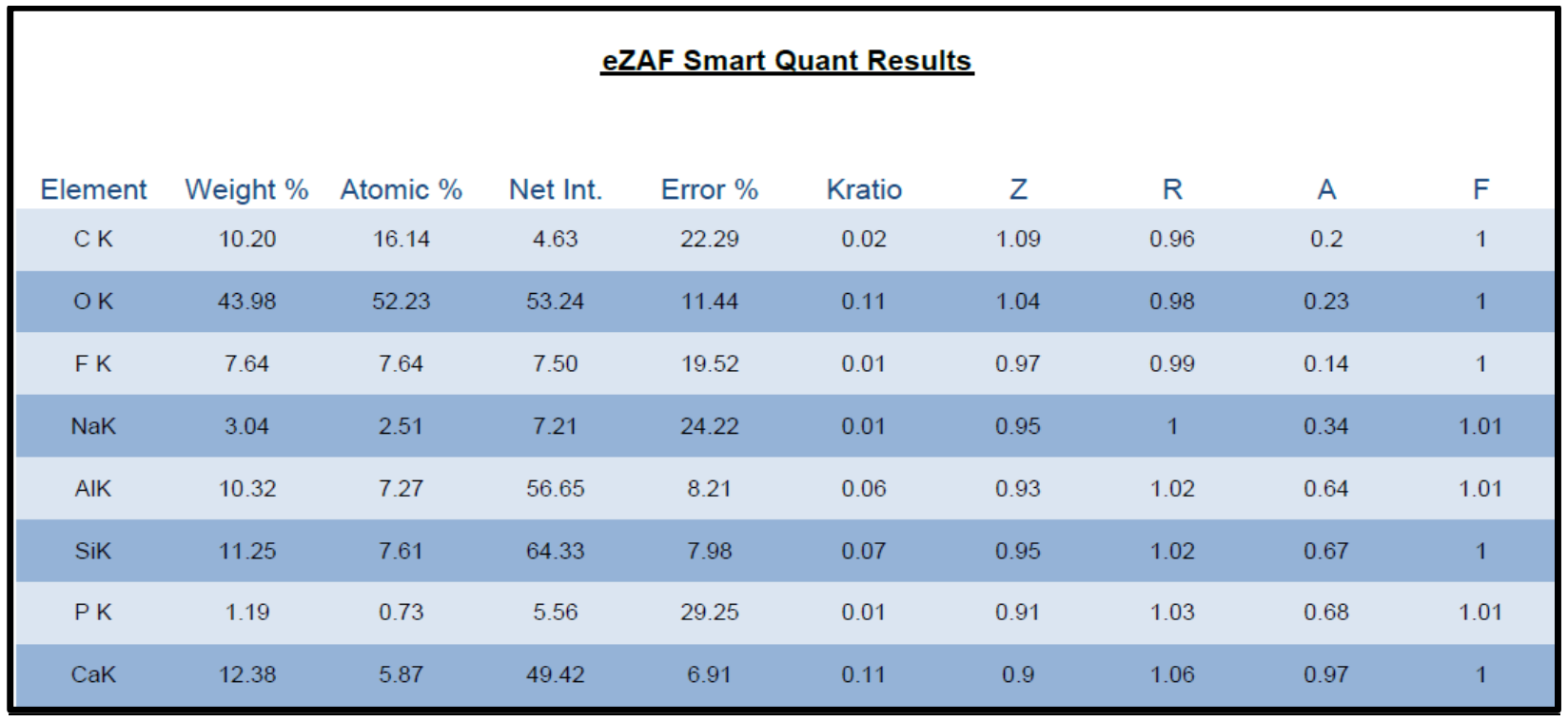

Figure no 7. Chart showing elemental analysis (Group I - Type II GIC) 


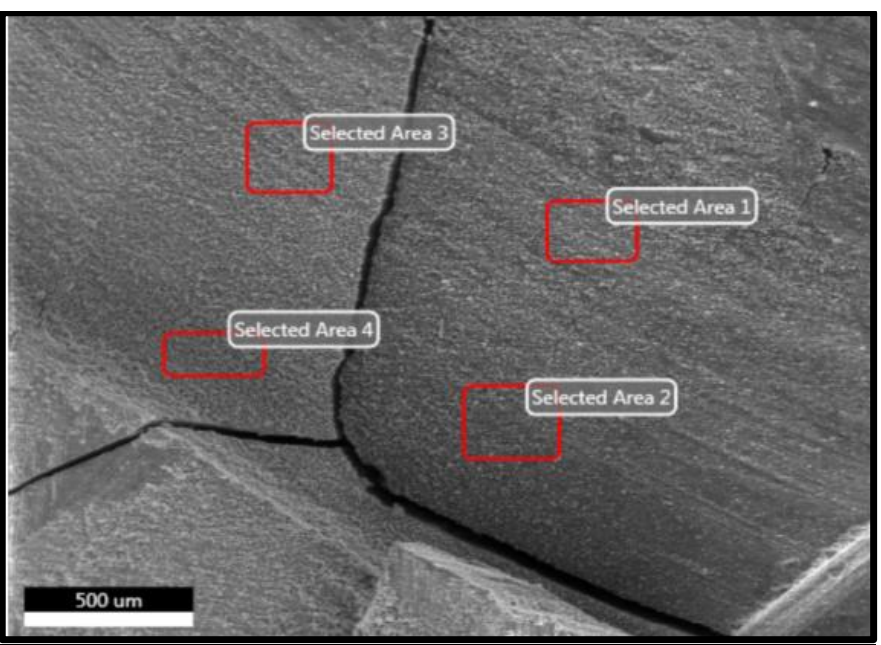

Figure no 8.SEM image at x100 magnification (Group II -CHXHMP-GIC)

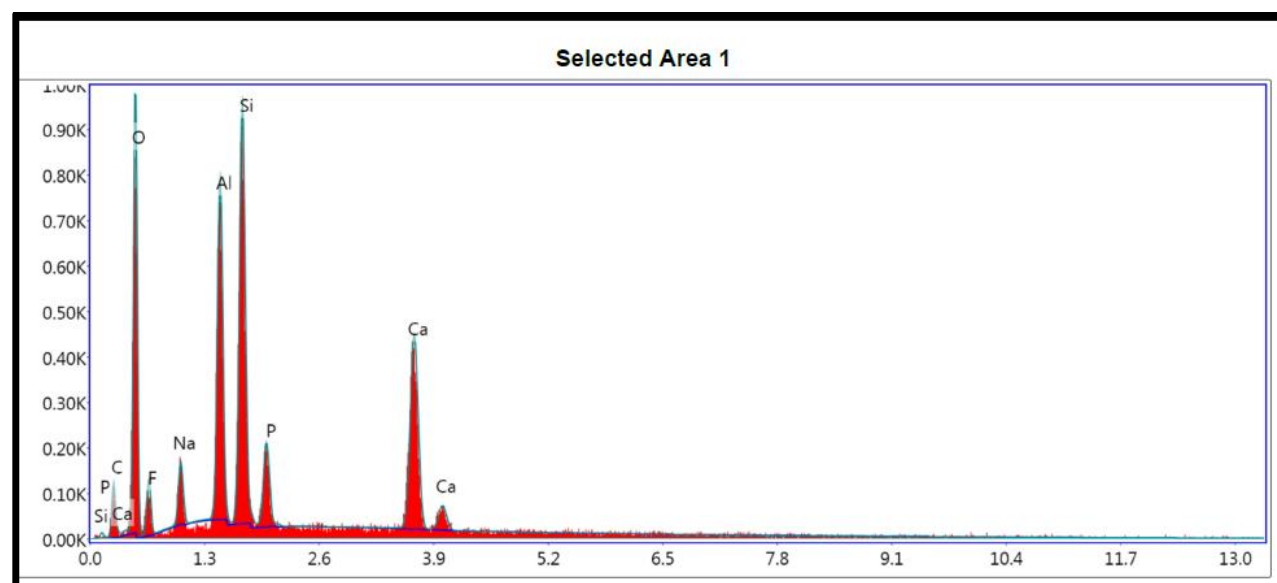

ec: 30.00 Cnts 0.000 keV Det: Octane Plus Det

Figure no 9.EDS analysis (Group II -CHXHMP-GIC)

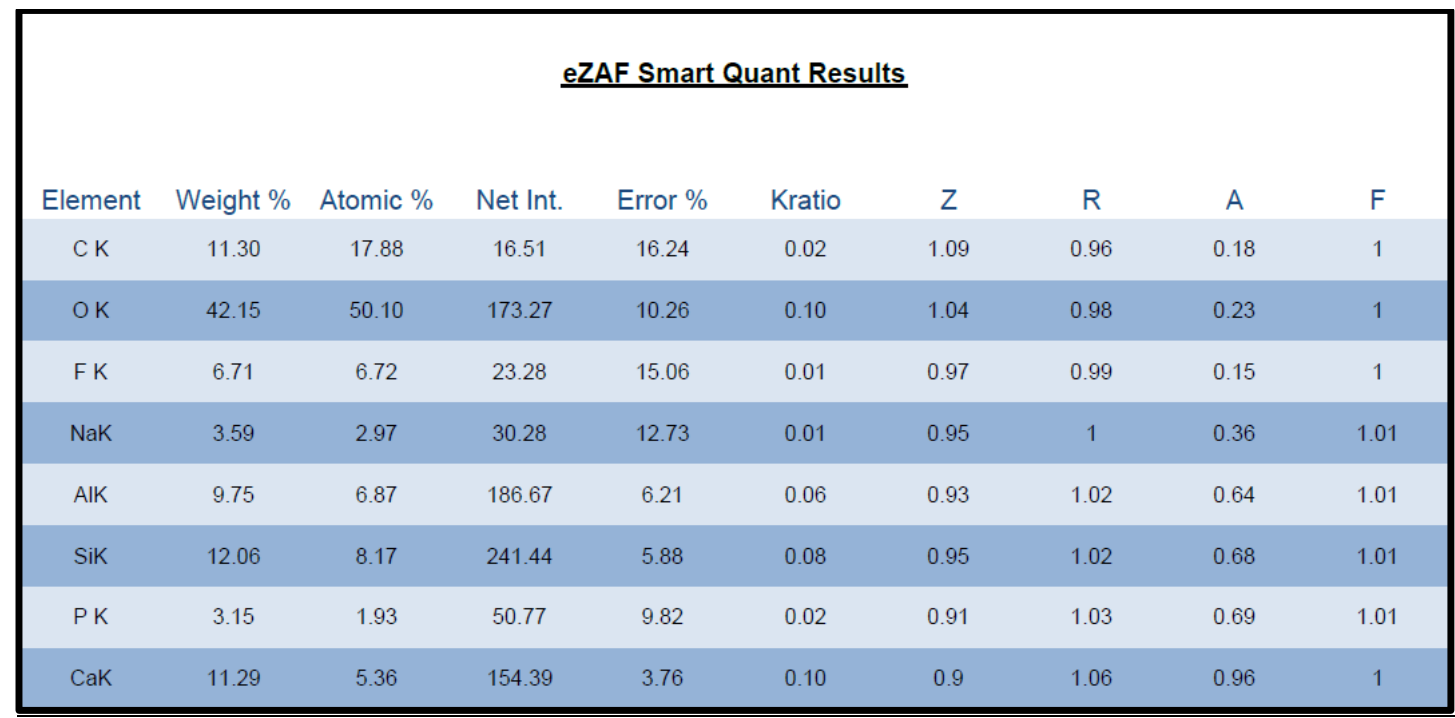

Figure no 10. Chart showing elemental analysis (Group II -CHXHMP-GIC) 


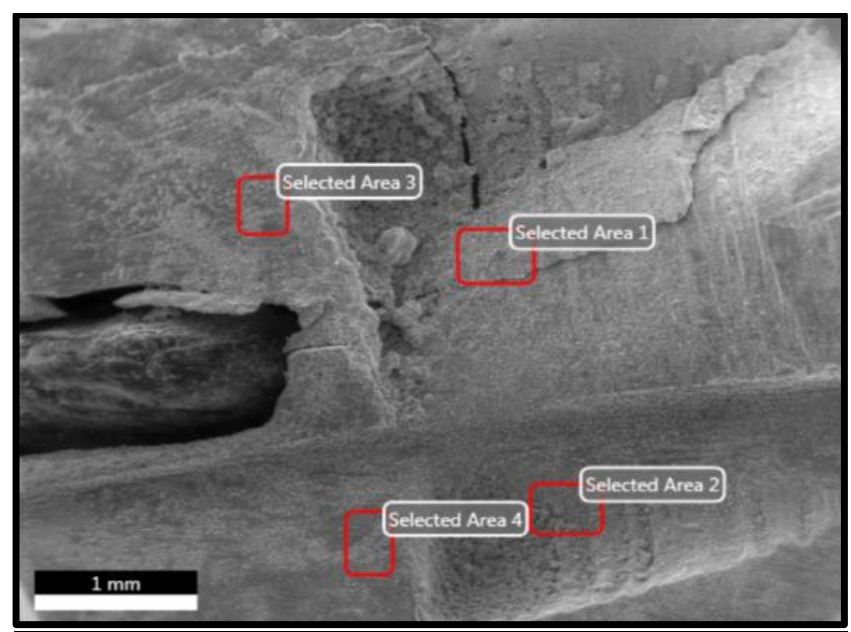

Figure no 11.SEM image at x100 magnification (Group III -control)

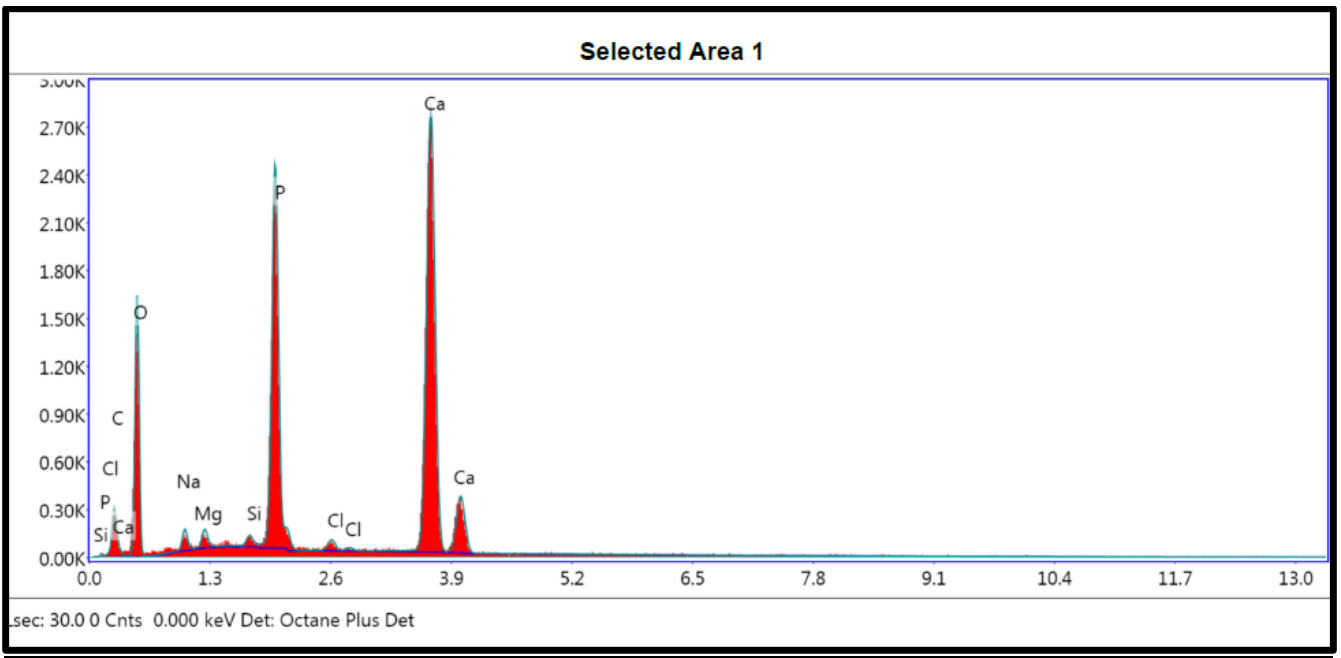

Figure no 12.EDS analysis (Group III -control)

\begin{tabular}{|c|c|c|c|c|c|c|c|c|c|}
\hline \multicolumn{10}{|c|}{ eZAF Smart Quant Results } \\
\hline Element & Weight \% & Atomic \% & Net Int. & Error \% & Kratio & Z & $\mathrm{R}$ & A & $\mathrm{F}$ \\
\hline $\mathrm{CK}$ & 7.78 & 13.52 & 32.90 & 14.76 & 0.02 & 1.1 & 0.94 & 0.22 & 1 \\
\hline $\mathrm{OK}$ & 44.95 & 58.64 & 291.54 & 10.57 & 0.07 & 1.06 & 0.96 & 0.15 & 1 \\
\hline NaK & 1.76 & 1.60 & 32.32 & 13.00 & 0.01 & 0.96 & 0.99 & 0.32 & 1 \\
\hline MgK & 0.90 & 0.77 & 30.96 & 13.72 & 0.00 & 0.98 & 1 & 0.46 & 1.01 \\
\hline SiK & 0.36 & 0.27 & 19.60 & 19.53 & 0.00 & 0.96 & 1.01 & 0.74 & 1.02 \\
\hline $\mathrm{PK}$ & 13.96 & 9.41 & 675.73 & 3.48 & 0.11 & 0.93 & 1.02 & 0.84 & 1.02 \\
\hline $\mathrm{CIK}$ & 0.45 & 0.27 & 20.43 & 22.64 & 0.00 & 0.9 & 1.03 & 0.87 & 1.04 \\
\hline CaK & 29.83 & 15.53 & $1,000.56$ & 2.07 & 0.26 & 0.91 & 1.05 & 0.97 & 1 \\
\hline
\end{tabular}

Figure no13.Chart showing elemental analysis (Group III -control) 


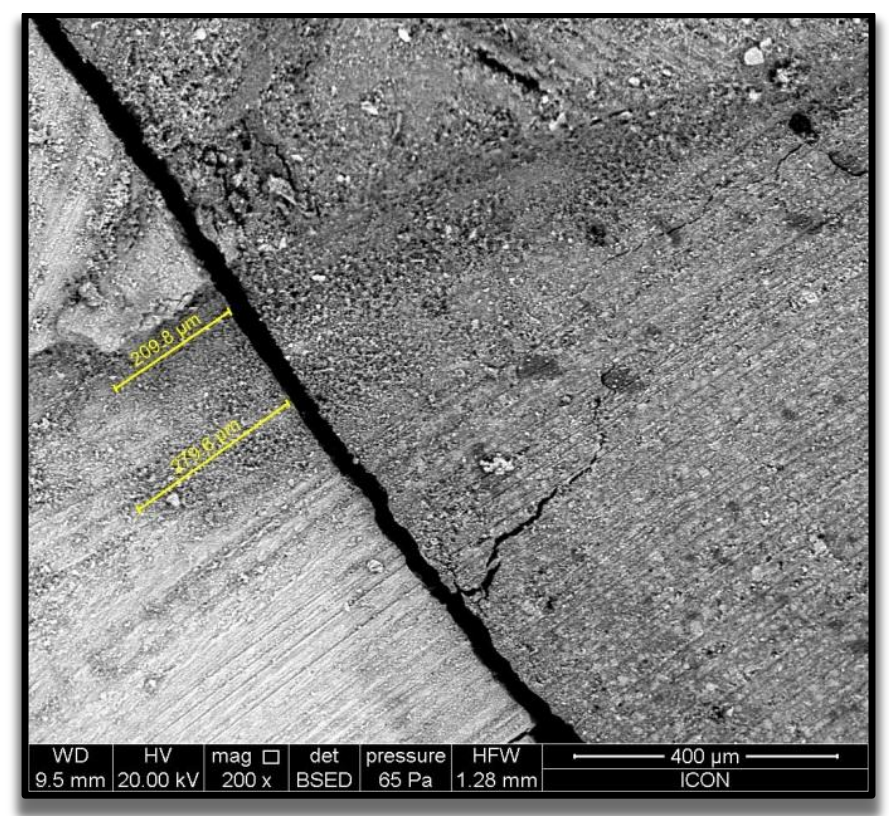

Figure no 14. SEM image showing depth of fluoride penetration for Group I- GIC

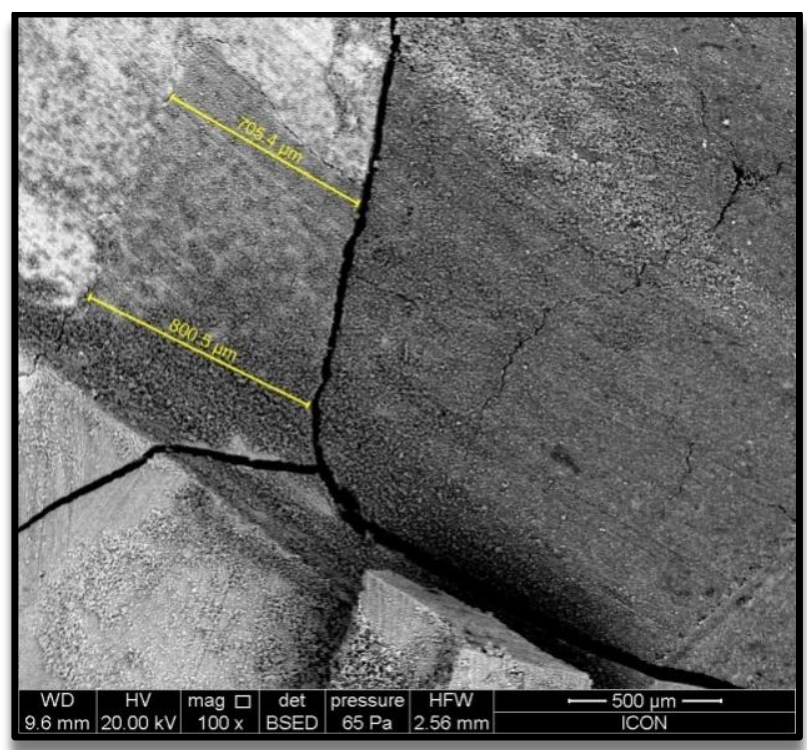

Figure no 15. SEM image showing depth of fluoride penetration for Group II- CHXHMP-GIC 


\section{A)}

$$
\mathrm{Ca}_{10}\left(\mathrm{PO}_{4}\right)_{6}(\mathrm{OH})_{2} \quad \mathrm{Ca}_{10}\left(\mathrm{PO}_{4}\right)_{6} \mathrm{~F}_{2}
$$

(Hydroxyapatite) (Fluorapatite)

B)

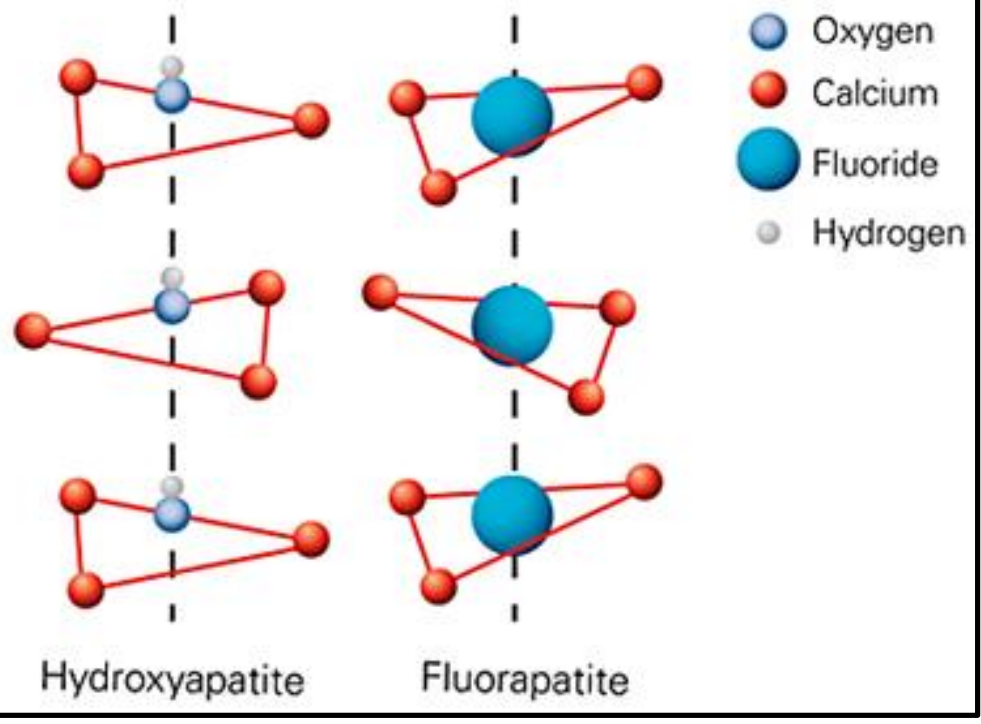

Figure 16.Fluorapatite Formation (A) Fluoride ions (F-) replace hydroxyl ions $(\mathrm{OH}-)$ in hydroxyapatite to form fluorapatite in the tooth enamel. (B) A portion of the apatite crystal lattice is depicted showing the replacement of hydroxide for fluoride.

Table no.1 Table showing amount of fluoride (in atomic \%)

\begin{tabular}{|c|c|c|}
\hline Sample no. & $\begin{array}{c}\text { Group I } \\
\text { (Type II GIC) } \\
\text { Atomic wt\% }\end{array}$ & $\begin{array}{c}\text { Group II } \\
\text { ( CHXHMP-GIC) } \\
\text { Atomic wt\% }\end{array}$ \\
\hline 1 & 7.64 & 6.65 \\
\hline 2 & 7.70 & 6.75 \\
\hline 3 & 7.62 & 6.73 \\
\hline 4 & 7.58 & 6.68 \\
\hline 5 & 7.63 & 6.71 \\
\hline 6 & 7.67 & 6.74 \\
\hline Mean & 7.64 & 6.71 \\
\hline
\end{tabular}

\section{Conclusion:-}

Under the limitations of the study, the following conclusions can be drawn.

1) Type II GIC released more fluoride as compared to CHXHMP-GIC nanoparticles.

2) Depth of fluoride penetration in dentin was more with nanoparticles CHXHMP- GIC as compared with Type II GIC. 


\section{References:-}

1. Mount GJ, Ngo H. Minimal intervention: a new concept for operative dentistry. Quintessence Int Sept 2000; 31(8): 527-33.

2. Peters MC, McLean ME. Minimally invasive operative care. II. Contemporary techniques and materials: an overview. J Adhes Dent Spring 2001; 3(1): 17-31.

3. Herrera M, Castillo A, Baca P, Carrión P. Antibacterial activity of glass-ionomer restorative cements exposed to cavity producing microorganisms. Oper Dent 1999; 24: 286-91.

4. Moshaverinia A, Chee WW, Brantley WA et al. Surface properties and bond strength measurements of Nvinylcaprolactam (NVC) containing glass-ionomer cements. J Prosthet Dent Mar 2011; 105(3):185-93.

5. Ten Cate JM, Van Duinen RN. Hypermineralization of dentinal lesions adjacent to glass-ionomer cement restorations. J Dent Res Jun 1995; 74(6):1266-71.

6. Wang Z, Shen Y, Haapasalo M. Dental materials with antibiofilm properties. Dent Mater 2013; 30: e1-e16.

7. Hook et al. Development of a novel antimicrobial-releasing glass ionomer cement functionalized with chlorhexidinehexametaphosphate nanoparticles. Journal of Nanobiotechnology 2014; 12: 3.

8. HK, Lam WT, Smales RJ. Fluoride release, weight loss and erosive wear of modern aesthetic restoratives. $\mathrm{Br}$ Dent J. 1999 Sep 11;187(5):265-70.

9. Dunne SM, Goolnik JS, Millar BJ, Seddon RP. Caries inhibition by a resin-modified and a conventional glass ionomer cement, in vitro. J Dent. 1996 Jan-Mar;24(1-2):91-4.

10. Pilly V et al Protection of tooth structure by chlorhexidine and natural polyphenols: a review Braz Dent Sci2012;15 (4)

11. Sanders BJ, Gregory RL, Moore K, Avery DR: Antibacterial and physical properties of resin modified glassionomers combined with chlorhexidine. J Oral Rehabil 2002, 29:553-558.

12. Jedrychowski JR, Caputo AA, Kerper S. Antibacterial and mechanical properties of restorative materials combined with chlorhexidines. J Oral Rehabil. 1983 Sep; 10(5):373-81.

13. Tüzüner T, Kuşgöz A, Er K, Taşdemir T, Buruk K, Kemer B. Antibacterial activity and physical properties of conventional glass-ionomer cements containing chlorhexidinediacetate/cetrimide mixtures. J EsthetRestor Dent. 2011 Feb; 23(1):46-55.

14. Hoszek A, Ericson D. In vitro fluoride release and the antibacterial effect of glass ionomers containing chlorhexidinegluconate. Oper Dent. 2008 Nov-Dec;33(6):696-701.

15. Barbour ME, Maddocks SE, Wood NJ et al. Synthesis, characterization, and efficacy of antimicrobial chlorhexidinehexametaphosphate nanoparticles for applications in biomedical materials and consumer products. Int J Nanomedicine 2013; 8: 3507-3519.

16. Gerould $\mathrm{CH}$. Electron microscope study of the mechanism of a fluorine deposition in teeth. Journal of Dental Research 1945; 24: 223-233.

17. Duschner H, Götz H, Ogaard B. Fluoride-induced precipitates on enamel surface and subsurface areas 208visualized by electron microscopy and confocal laser scanning microscopy. European Journal of Oral Science 1997; 105: 466-472

18. Harding AM, Zero DT, Featherstone JD, McCormack SM, Shields CP, Proskin HM. Calcium fluoride formation on sound enamel using fluoride solutions with and without lactate. Caries Research 1994; 28: 1-8.

19. Weidlich P, Miranda LA, Maltz M, Samuel SM. Fluoride release and uptake from glass ionomer cements and composite resins. Braz Dent J. 2000;11(2):89-96.

20. Aboush YEY, Torabzadeh H. Fluoride release from tooth-colored restorative materials: a 12-month report. Canadian Dent Assoc J 1998; 64(8):561-564, 568

21. Yohe, B. Tooth Paste. ChemMatters, 1986;4 (1):12-13

22. http://www.dentalcare.com/en-US/dental-education/continuing education/ce410/ce410.aspx?ModuleName=coursecontent $\&$ PartID=2\&SectionID $=1$

23. Peter C. Moon. Review of Matrix Metalloproteinases' Effect on the Hybrid Dentin Bond Layer Stability and Chlorhexidine Clinical Use to Prevent Bond Failure. The Open Dentistry Journal 2010; 4:147-152.

24. S. MostafaMousavinasab and Ian Meyers. Fluoride Release and Uptake by Glass Ionomer Cements, Compomer and Giomers. Research Journal of Biological Sciences.2009:4(5):609-616. 